Bundesgesundheitsbl 2015 · 58:1126-1128

DOI 10.1007/s00103-015-2230-6

Online publiziert: 28. September 2015

(c) Springer-Verlag Berlin Heidelberg 2015

Mitteilungen des Arbeitskreises Blut des Bundesministeriums für Gesundheit

\title{
Bewertung von Apherese- und Pool-Thrombozytenkonzentraten
}

satz zu Vollblutfiltration der Hb-Gehalt des EK um ca. $10 \%$ niedriger. Die Menge an gleichzeitig gewonnenem Plasma ist jedoch höher.

- ATK werden derzeit (2014) mehrheitlich in Plasma suspendiert (84\%), PTK mehrheitlich in Additivlösung (79\%). chen Verfahren hergestellt: Bei Apherese-Thrombozytenkonzentraten (ATK) wird die für das Präparat erforderliche Thrombozytenmenge mittels Apherese von einem Spender gewonnen. Bei einer ATK-Spende werden regelhaft zwei oder sogar drei ATK hergestellt. Für die Herstellung von Pool-Thrombozytenkonzentraten (PTK) werden die Thrombozyten aus vier Blutgruppen-gleichen Vollblutspenden zusammengeführt. Für beide Präparate gelten dieselben Spezifikationen. Im Jahr 2013 erfolgte die Versorgung mit $61,5 \%$ ATK und 38,5\% PTK.

Aktuell gibt es eine intensive Diskussion, ob die beiden Herstellungswege zu gleichwertigen Produkten führen. Hierbei ist insbesondere die Frage der Relevanz der erhöhten Spenderexposition bei PTK mit Blick auf Infektionsrisiken von Bedeutung. Diese Debatte muss auch mit Blick auf die therapeutischen Anforderungen, die bestehenden und künftigen Versorgungsstrukturen, den Blickwinkel der Spender und rechtliche Rahmenbedingungen erfolgen.

Der Arbeitskreis Blut kommt zu folgenden Ergebnissen:

\section{Herstellung}

- Die Herstellungsverfahren für ATK und PTK sind in Deutschland seit Jahren etabliert.

- PTK werden in Deutschland ausschließlich mit der so genannten „Buffy Coat" Methode hergestellt.

- Die Herstellung von PTK ist aktuell nur bei einer EK-Inline-Filtration möglich. Bei dieser ist im Gegen-

\section{Therapie}

- Ungefähr $75 \%$ der TK werden bei Patienten in der Hämatologie/Onkologie eingesetzt. Eine Blutungsneigung ist neben den absoluten Thrombozytenwerten entscheidend von Patienten-abhängigen Faktoren beeinflusst.

- Patienten mit Antikörpern gegen Leukozyten- oder Thrombozytenantigene (HLA- bzw. HPA-Antikörper) benötigen zwingend typisierte TK von ausgewählten Spendern. Die Bereitstellung von typisierten TK ist nur durch entsprechend ausgewählte ATK-Spenden möglich.

- Die Therapie soll entsprechend den Empfehlungen der Querschnittsleitlinien erfolgen. Weiterhin sind auch aktuell publizierte Erkenntnisse zu berücksichtigen.

- Der optimale Thrombozytengehalt der Präparate ist aktuell Gegenstand von Studien.

\section{Wirksamkeit}

Hinsichtlich der klinischen Wirksamkeit liegen keine prospektiv-randomisierte Studien zu einer gleichwertigen oder unterschiedlichen therapeutischen Wirksamkeit von PTK (buffy coat) oder ATK vor.

\section{Risiken für Empfänger}

- Die Anwendung von TK ist sicher. Sie führt nur in seltenen Fällen zu schwerwiegenden Nebenwirkungen beim Patienten.
- Zur Abschätzung von Risiken stehen Hämovigilanzdaten, epidemiologische Daten, Studien und mathematische Modelle zur Verfügung.

- Anhand der Hämovigilanzdaten zeigt sich kein Unterschied in der Häufigkeit berichteter viraler oder bakterieller Infektionen zwischen ATK und PTK: HIV- Transmissionen durch TK wurden in dem Zeitraum 1997-2012 nicht gemeldet, die letzten $\mathrm{HCV}$ Transmissionen nach TK-Gabe wurden in den Jahren 1998/99 gemeldet. Zwischen 1997 und 2012 wurden insgesamt 5 HBV-Übertragungen durch ATK gemeldet, zuletzt 2012, keine durch PTK. Bei den gemeldeten bakteriellen Übertragungen lag die Häufigkeit der Meldungen zwischen 1997 und 2011 bezogen auf 1 Mio. TK bei 6 jeweils für ATK und PTK.

- Die geschätzten Infektionsrisiken für HIV-, HCV- und HBV-Infektionen sind anhand von mathematischen Modellen basierend auf Meldedaten von Blutspendern unterschiedlich: Nach aktuellen Modellierungen unter den gegebenen Annahmen ist das Risiko eines unerkannt infektiösen Produktes bei PTK um den Faktor 1,5 höher für HCV- Infektionen. Für HBV ist das Risiko einer unerkannt infektiösen Spende bei ATK um den Faktor 1,6 höher. Für HIV sind die Risikounterschiede nicht signifikant.

- Die Risikounterschiede zwischen ATK und Vollblutspenden resultieren aus den unterschiedlichen Spenderpopulationen einerseits und den kürzeren Spendeintervallen bei ATK andererseits.

- Würde man alle tatsächlich gemeldeten Spendeintervalle der positiv getesteten Spender im mathematischen Modell fiktiv auf mindestens $30 \mathrm{Ta}$ - 
ge erhöhen, verschieben sich die Risikounterschiede zugunsten der ATK: Das Risiko eines unerkannt infektiösen Produktes ist für PTK um den Faktor 1,65 höher für HIV- Infektionen und um den Faktor 3,9 höher für HCV Infektionen. Für HBV wären die Risikounterschiede unter diesen Annahmen nicht signifikant.

- Die mathematischen Modelle können immer nur ein gemitteltes Risiko schätzen, das punktuelle Risiko für den einzelnen Transfusionsempfänger ist weitaus schwerer zu quantifizieren.

- Die beobachteten Infektionshäufigkeiten bei den getesteten Infektionen sind in verschiedenen Spenderpopulationen unterschiedlich. Es gibt Unterschiede in der Infektionshäufigkeit zwischen Stadt- und Landbevölkerung, verschiedenen Altersgruppen und den Geschlechtern. Die geschätzten Risikodifferenzen zwischen diesen Spenderpopulationen („,intrinsisches Risiko") liegen zum Teil über den Risikodifferenzen, die für beide TK-Arten geschätzt werden.

- Für unbekannte Erreger ist die Risikoabschätzung mit deutlich mehr Unsicherheiten behaftet, da weder die Erregereigenschaften noch die Verteilung in der Spenderpopulation bekannt sind. Grundsätzlich könnte die Tatsache, dass PTK aus mehreren Spenden hergestellt werden (erhöhte Spenderexposition) zu einem höheren Infektionsrisiko durch PTK beitragen, maximal bis zu dem PoolingFaktor (aktuell 4). Jedoch könnte ein Poolen von Spenden z. B. durch die Reduktion der Infektionsdosis oder die Transfusion von protektiven Antikörpern auch zu einem geringeren Infektionsrisiko bei PTK führen.

- Bei nicht-infektiösen unerwünschten Reaktionen gibt es Unterschiede in Abhängigkeit von den verwendeten Suspensionsmedien. TK in Additivlösung verursachen seltener nicht schwerwiegende unerwünschte Transfusionsreaktionen als TK in Plasma. Darüber hinaus gibt es keine Daten, die auf Unterschiede in der Häufigkeit und/oder Schwere nichtinfektiöser Reaktionen zwischen ATK und PTK hinweisen.
- Die Möglichkeiten der Pathogeninaktivierung wurden bei den Betrachtungen außer Acht gelassen. Eine Stellungnahme zu den Pathogeninaktivierungsmethoden von Blutprodukten befindet sich in Vorbereitung.

\section{Spenderperspektive}

- Beide Spendeverfahren beinhalten wenige Risiken für die Spender. Bei Apheresen sind zusätzlich zu den Punktions- und Kreislaufrisiken die möglichen für das Spendeverfahren typischen Nebenwirkungen zu beachten.

- Da PTK aus Vollblutspenden hergestellt werden, die primär der Herstellung von EK dienen, entsteht durch die PTK-Herstellung kein zusätzliches Spenderrisiko.

- ATK-Spenden werden ausschließlich für die TK-Herstellung durchgeführt und die möglichen Nebenwirkungen sind somit der Herstellung des ATK zuzuordnen.

- Werden ATK aus Multikomponentenspenden hergestellt, sind die Risiken für den Spender ebenfalls nicht allein dem TK zuzuordnen.

\section{Versorgungsstruktur}

- Eine Verschiebung der Herstellungskapazitäten zugunsten von ATK bis hin zur Vollversorgung wäre möglich. Hierzu müssten entweder die Spendefrequenzen oder die Anzahl der Spender erhöht werden oder eine Kombination aus beiden Möglichkeiten erfolgen. Alternativ könnten von entsprechend selektierten Spendern auch mehr ATK aus einer Spende gewonnen werden.

- Eine Verschiebung der Herstellungskapazitäten zugunsten von PTK wäre ebenfalls möglich. Kleinere Spendeeinrichtungen mit weniger als 50.000 Spenden jährlich könnten jedoch nicht selbstversorgend mit TK werden, insbesondere bei Rhesus-D-negativen Blutgruppen. Dies betrifft nahezu alle universitären und staatlichkommunalen Spendeeinrichtungen. Zusätzlich könnten ein vermindertes Spenderaufkommen oder Fluktuationen bei den Vollblutspenden Probleme bei der Versorgung aufwerfen.
Zur Kompensation wären vermutlich größere Strukturänderungen erforderlich.

- Für die Versorgung der aus immunologischen Gründen Therapie-refraktären Patienten ist eine kliniknahe Versorgung mit typisierten ATK erforderlich. Dies setzt einen typisierten Spenderstamm mit Spendern voraus, die regelmäßig Apheresespenden in entsprechend ausgestatteten Zentren leisten.

- Aktuell werden ATK und PTK unterschiedlich erstattet. Die aktuelle Situation, in der ATK z. T. nur unter Vorbehalt erstattet werden, führt zu starker Verunsicherung und gefährdet möglicherweise die bestehende Versorgungsstruktur.

- Eine Veränderung der aktuellen Versorgungsstruktur könnte auf die Risiken beider Produkte und auf die Struktur der Spendeeinrichtungen insgesamt Auswirkungen haben. Eine gesicherte Versorgung könnte bei Konzentration auf eine Herstellungsart gefährdet sein.

Der AK Blut plädiert daher für den Erhalt der dualen Versorgungsstruktur mit PTK und ATK, um die sichere und gesicherte Versorgung der Bevölkerung aufrecht zu erhalten. Zusätzlich ist für die Versorgung von immunisierten Patienten mit HLA- bzw. HPA-typisierten ATK zwingend ein hinreichend typisierter aktiver Spenderstamm und Expertise in Thrombozytapheresen erforderlich.

Die Hämovigilanzdaten liefern keine Evidenz für eine unterschiedliche Risikoeinschätzung für beide Präparate. Hinsichtlich der Infektionsrisiken zeigt sich eine Differenz in der mathematischen Schätzung für bekannte Erreger.

Bei der Bewertung der Unterschiede bei den geschätzten Infektionsrisiken wurde berücksichtigt, dass diese im Bereich der Schwankungsbreite von Infektionsrisiken bei Spenden humanen Ursprungs liegen („,intrinsische Risiken“ $z$. B. zwischen verschiedenen Spenderpopulationen (abhängig z. B. vom Geschlecht, Wohnregion der Spender)). Gleichzeitig liegen keine Hinweise auf unterschiedliche Risiken in den Hämovigilanzmeldungen vor. Dies führt zu der Einschätzung, 
dass beide Produkte als wirksam und sicher anzusehen sind.

Die beiden Produkte sind jedoch nicht gleichartig und sind damit nicht ohne weiteres austauschbar. Es obliegt dem behandelnden Arzt im Rahmen seiner Therapieverantwortung, die entsprechend geeigneten Produkte auszuwählen.

An einzelnen Punkten gibt es Handlungsoptionen, um die Präparate zu optimieren: So zeigen die Hämovigilanzdaten, dass eine Suspension in Additivlösung günstig ist. Zusätzlich kann zur Reduktion des geschätzten Infektionsrisikos eine Verlängerung des Mindest-Spendenintervalls in den Hämotherapierichtlinien für ATK-Spenden für die Regelversorgung diskutiert werden. Bei den Therapieempfehlungen sollten bei entsprechend geeigneten Patienten die neueren Erkenntnisse zu prophylaktischen TK-Transfusionen berücksichtigt werden. Mit Blick auf die aktuelle Vergütungssituation ist die sachgerechte Abbildung beider Präparate im DRG-System notwendig, um negative Auswirkungen auf die Versorgungsstruktur zu verhindern.

Ausführliche wissenschaftliche Erläuterungen zu dieser Stellungnahme finden sich in einem separaten Manuskript.

Für den Arbeitskreis Blut

Dr. R. Offergeld, Vorsitzende 